\title{
Metáfora interactiva en ESPACIOS FICTICIOS: EXPRESIÓN ESTÉTICA DE CONTENIDOS EN UNA APLICACIÓN HIPERMEDIA
}

\author{
INTERACTIVE METAPHOR IN FICTITIOUS SPACES
}

Por:

\author{
Javier M. Reyes Vera \\ Profesor Departamento de Diseño \\ Universidad del Valle. Cali, Colombia \\ javier.reyes@correounivalle.edu.co
}

Resumen: Últimamente el Html5 ha cobrado gran auge debido a la versatilidad con la que se puede presentar la información a los usuarios y el despliegue estético que es evidente en sus apuestas, mediadas por transiciones, uso de paradigmas diversos de interacción (como pestañas, migración de fondos, texturas, valores tonales...), manejo de estilos tipográficos y de botones que le permiten al usuario del común tener una relación mucho más sensible relacionándolo con lo que se conoce el día de hoy como Net-Art. Sin embargo ese despliegue de formas, texturas y transiciones se hace posible gracias al manejo adecuado de aspectos técnicos y tecnológicos que se logran por el trabajo integral de un grupo en el que cada uno de los actores desempeña un rol importante. Es por eso que se logra un resultado en el que se conjugan aspectos de orden etnográfico, de interfaz y estéticos en un producto cuidadosamente pensado en sus detalles. El presente artículo muestra los elementos conceptuales que permitieron una expresión estética de los contenidos de la Hipermedia Salsa Barrio Cultura ${ }^{1}$ desde la perspectiva del diseño de interfaces.

Palabras Clave: Adaptatividad, Diseño de Interfaces, Espacios Ficticios, Hipermedia, Usabilidad. 
Abstract: Recent years Html5 has become in a new purpose because of the versatility in the presentation of diverse information, their aesthetic displays, using of diverse interaction paradigms (such as tabs, textures, tonal values...), management of type styles and buttons that allow common users to have a sensible relationship. It is known today as Net-Art. But this set of shapes, textures and transitions is possible by proper use of technical and technological aspects, but is important for a team where all of the actors play a relevant role. For this reason we can work with ethnographical aspects, interface issues and aesthetic in a product thinking about each detail. This article presents the conceptual elements that allowed an aesthetic expression of the contents of the Hipermedia Salsa Barrio Cultura from the interface design perspective.

Keywords: Adaptivity, Interface Design, Fictional Spaces, Hypermedia, Usability.

\section{Introducción}

La Hipermedia Salsa Barrio Cultura es un proyecto de creación original aprobado en la convocatoria de Investigación y Creación de la Vicerrectoría de investigaciones de la Universidad del Valle en el 2012. Dicho proyecto es la evolución conceptual y de contenidos de su antecesor denominado Multimedia Cali Cultura Salsera ${ }^{\circledR}$ (URL 1) también desarrollado en el marco de una convocatoria anterior.

El proyecto se formalizó en conjunto con dos grupos de investigación de la Universidad del Valle: el grupo Palo de Mango de la Escuela de Comunicación Social y el grupo Camaleón de la Escuela de Ingeniería de Sistemas y computación de la Universidad del Valle. Este proyecto estuvo bajo la dirección del profesor, investigador y antropólogo Alejandro Ulloa Sanmiguel, reconocido por sus investigaciones de la cultura salsera y autor de varios textos académicos relacionados con el tema.

Este proyecto de creación aspira a divulgar entre una comunidad heterogénea, primordialmente de melómanos, coleccionistas e investigadores, un conjunto de elementos que apoyen conceptualmente el estudio de la Salsa como fenómeno cultural y que puedan ser consultados en diversas partes del mundo. Para ello se pensó en el recurso que ofrece la hipermedia y la web, asimismo el uso de diversidad de dispositivos móviles que hoy son de uso común. Esto permite ampliar el espectro del público y superar las limitaciones técnicas y tecnológicas que se presentaron en la implementación del proyecto anterior (desarrollado en Adobe Flash $®)$.

A continuación se expondrá un conjunto de elementos conceptuales que permitieron abordar el proyecto desde el punto de vista técnico, tecnológico y de interfaz, haciendo énfasis en las posibilidades y limitaciones que tiene el uso del Html5 en la concepción de lo que para el proyecto se han denominado espacios ficticios. De esta manera, el presente artículo está organizado siguiendo la siguiente estructura: primero se presenta la evolución de las interfaces; posteriormente se ofrece una reflexión relacionada con los paradigmas de interacción; luego los usuarios y sus características, la adaptatividad presente en la Hipermedia, la conceptualización de la estructura narrativa a partir de las metáforas, los lugares ficticios y la hipertextualidad. Al final, las conclusiones que son el punto de partida y de reflexión de éste y de futuros proyectos de creación. 


\subsection{La mutación de las interfaces: de la ausencia a la presencia}

Ya en el año 1945, Bush proponía los computadores como una suerte de asistentes documentales que facilitaban el almacenamiento e interrelación de documentos. Imaginó un dispositivo que extendía la capacidad de memoria humana y permitía guardar información de todo tipo y consultarla con rapidez: lo que bautizó como Memex ${ }^{2}$. Estableció las bases principales de lo que posteriormente se llamaría hipertexto (Nelson ,1945).

En los primeros años 50 las computadoras eran una máquinas pensadas para matemáticos y científicos cuyo principal objetivo de creación era la confiabilidad y la rapidez en los cálculos (Shackel, 1997), por tal razón su uso estaba restringido a un personal muy especializado y con un nivel de formación académica específico.

Posterior a ello, al inicio de los años 60 un psicólogo llamado Frederic Bartlett definió las siguientes tendencias que serían el punto de referencia para los años venideros (Bartlett, 1962 citado en Carocci, 2011):

- Crecimiento del aislamiento físico de los individuos

- Necesidad mayor de comunicación mediada por la tecnología

- Reducción de la carga de trabajo física

- Incremento de la carga de trabajo mental

- Integración del trabajo de más personas en el trabajo de una única persona

- Presentación de estímulos multimodales

- Énfasis en el crecimiento de la actividad de tomar decisiones

Con base en los anteriores elementos observamos que existía un crecimiento gradual respecto al interés expedito de brindar gran relevancia a los aspectos relacionados con la interfaz y la interacción que implica acciones cognitivas y de comunicación que van más allá de la simple interacción física; aquí se dibuja una propuesta que permite ampliar espectros relacionados con la carga de trabajo de los usuarios, la automatización, el manejo de roles y la facilidad de comunicación que ofrecen las interfaces (Licklider \& Carl, 1990, Engelbart, 1992).

Grudin (1990) explica la dificultad que se tiene en esos primeros estadios para hablar del usuario y de interfaces, ya que estas últimas eran consideradas el hardware por sí mismo; en tal sentido los usuarios debían adaptarse a las prestaciones que ofrecían las aplicaciones y el concepto de interactividad estaba limitado al aprendizaje en el uso especializado de las interfaces o aplicaciones. En esa década (años 60-70) aparecen las denominadas macrocomputadoras -mainframes- que son dirigidas a profesionales en el procesamiento de datos. Sin embargo, los usuarios difícilmente pueden acceder debido a los altos costos, latencias y poca flexibilidad de estos aparatos (Shakel, 1997). 
En estos incipientes inicios algunos investigadores encuentran opciones y posibilidades con el uso del computador, los científicos Danny Bobrow, Wallace Feurzeig y Seymour Papert (Feurzeing, 1966) crean el conocido lenguaje de programación LOGO que consistía en una tortuga que realizaba formas mediante instrucciones simples, esto demostró que los niños podían familiarizarse fácilmente con los lenguajes de programación sin ninguna formación especializada, idea que sería retomada por los pioneros de la masificación de los dispositivos con el objetivo de generalizar su uso a públicos de diversas índoles.

A finales de los años 70 y al inicio de los 80, empieza una masificación del computador personal dirigido a profesionales no informáticos pero que aún así debían realizar programación sobre las máquinas, por lo tanto no eran usuarios del común sino que seguían siendo especializados, además se popularizó el uso de las redes informáticas lo que permitió la aparición del correo electrónico que se convirtió en una de las primeras herramientas de trabajo colaborativo asincrónico y que permitió romper paradigmas relacionados con la distancia geográfica y la inmediatez de la información.

Es ya propiamente en la década de los 80 cuando se populariza el uso de los computadores a un público del común, y se comienza a hablar de usabilidad (Shakel, 1997). Un ejemplo concreto es el lanzamiento de la WorkStation Star por parte de la empresa Xerox Parc ${ }^{\circledR}$ en el año 1981 (Carocci, 2011). Por otra parte, se estandarizaron los dispositivos de entrada y salida (pantalla, teclado, ratón, impresora). Además, Shneiderman (1987), estableció los fundamentos teóricos del concepto de manipulación directa y propuso las 10 reglas de oro (Shneiderman \& Plaisant, 2006); esta manipulación directa es evidente en el uso de ventanas que serían implementadas por Xerox en WIMP, comercializado por Apple y posteriormente imitado por Microsoft.

En la década siguiente surgen nuevas tecnologías que permiten la miniaturización de la informática, haciéndola no sólo más accesible en términos económicos sino más portable; su principal exigencia seguía siendo la usabilidad de las interfaces y se empiezan a desarrollar un conjunto de paradigmas que permiten una mejor utilización de las aplicaciones por medio del uso de metáforas. Se comienza a hablar de interfaces tipo WYSIWYG (WhatYou See Is WhatYou Get), donde el mayor exponente es el uso del ratón que maximiza la experiencia del usuario con el contenido de las aplicaciones.

Myers (1998) propone ese uso de la manipulación directa planteado por Shneiderman a partir del uso del ratón, interfaces con ventanas, procesadores de texto e hipertexto. 
Después de los 90 hasta la actualidad ocurren situaciones importantes que si bien no pueden circunscribirse a un periodo delimitado, se convierten en hitos que marcan las rutas de las prestaciones que nos permiten desarrollar productos digitales que están al alcance de todos. Estos hitos se evidencian en tres apartados diversos (Ribera, 2005):

\section{a. La Word Wide Web:}

Su aplicación primordial es la Internet, la cual permite generar interfaces basadas en el documento y no en la aplicación a la vez que rompe los límites entre información local y remota.

La web se convierte en un elemento clave en el desarrollo de la sociedad de la información, pues no sólo modifica radicalmente la conceptualización inicial que se tenía de los computadores sino que se inmiscuye en los aspectos prácticos de la vida cotidiana que van desde la investigación científica pasando por la educación (e-learning), la e-administración, la e-health, el e-bussines hasta el entretenimiento (e-entertaiment).

\section{b. Continuismo y crisis:}

Los aportes teóricos expuestos por Nielsen (1993) dan los inicios de un nuevo esquema que permite observar las interfaces desde una mirada más conceptual, encaminada al análisis detallado de las acciones de los usuarios y la funcionalidad que las mismas interfaces proveen. En este sentido la Ingeniería de Usabilidad, sumada a los métodos de evaluación de usabilidad permiten el crecimiento de la importancia del usuario en esa relación persona-computador.

Lo anterior sumando a la aparición y consolidación de las redes inalámbricas (bluettooth, wi-fi), el desarrollo de dispositivos móviles más pequeños y potentes, la consolidación de los portátiles, la integración de diversos dispositivos "programables" como algunos electrodomésticos, relojes, etc, generan un cambio particular en el paradigma de diseño y conceptualización de las interfaces que apoyan la consideración de una serie de elementos estéticos y permiten a los usuarios una mejor empatía en la interacción con la información.

\section{c. Computación Ubicua:}

Weiser (1991) se convierte en el pionero de este planteamiento a partir de la investigación desarrollada en Xerox Parc. Este paradigma indica que la computación ya no está anclada a un computador de escritorio sino que está diseminada en diversos computadores pequeños y especializados que están conectados en red. Los primeros experimentos del equipo de trabajo de Weiser hablan de un entorno ubicuo, en la actualidad la utilización de dispositivos móviles tales como tablets, smartphones, iPods, iPads etc, se ha masificado, a tal punto que es necesario considerar la relevancia que implica diseñar aplicaciones que estén en consecuencia con esta necesidad. El proyecto de creación Hipermedia Salsa Barrio Cultura precisamente busca que sea visualizada en estos diversos dispositivos para que los usuarios puedan disfrutar e interactuar con sus contenidos sin necesidad de estar en anclados a un computador de escritorio. 
Como vemos, no sólo ha mutado la concepción de interfaz sino que se han desarrollado a la par diversos métodos de creación que permiten al usuario estar cada vez más cerca de las aplicaciones. Además, es innegable la influencia que tiene el desarrollo técnico y tecnológico en la formalización y en la relación estética del usuario con las aplicaciones. Desde esa perspectiva, observamos como muchos diseñadores se ven obligados a tener en consideración esas nuevas tendencias para la creación de las interfaces por el simple hecho de que hay exigencias de tipo comercial que obligan a seguir una tendencia estética que empieza a ser recurrente en muchas aplicaciones.

\subsection{El paradigma mediado}

Una vez entendida la perspectiva relacionada con la influencia que tienen los avances tecnológicos respecto a la concepción estética, también debe serlo desde los paradigmas relacionados con la manipulación. De esta manera un usuario genera relaciones estrechas con los dispositivos en la medida que aumenta la frecuencia de uso. Esta relación inversamente proporcional es intangible pues está mediada por aspectos más cognitivos que físicos. Esa es la razón por la cual los usuarios como individuos desarrollamos diversos niveles de pericia en el uso de ciertas herramientas; son aspectos que si bien es cierto ayudan a comprender el fenómeno de interacción, no dan luces concretas que permitan abordar interfaces totalmente adaptativas a las necesidades particulares de los usuarios.

Vemos cómo los dispositivos enunciados en el apartado anterior cada día hacen más parte de nuestro entorno cotidiano, y así no queramos, presiones culturales, sociales y económicas nos obligan a aceptarlos, pues de no estar en sintonía con estas dinámicas, corremos el riesgo de ser aislados, no tener idea alguna de los cambios actuales y difícilmente sostener una charla en torno a los avances tecnológicos y los fenómenos que estos cambios generan a nivel social, cultural, económico y sicológico.

En concreto, los paradigmas están mediados no sólo por la disponibilidad de hardware sino de la relación que los individuos y los grupos sociales ejercen sobre estos elementos, haciendo que nos acerquemos o alejemos más de ciertos grupos que de no estar mediados por la tecnología no tendrían razón de ser $^{3}$.

En consecuencia con esa realidad, el proyecto Hipermedia Salsa Barrio Cultura hace uso de un conjunto de herramientas que permiten estar a la vanguardia de estas tendencias, sin demeritar la importancia que demandan la calidad de los contenidos relacionados con el tema de investigación que sustenta. En ese sentido, la hipermedia se convierte en una suerte de plataforma que, por sus cualidades, permite divulgar un conocimiento que, de otro modo, no llegaría de manera directa y podría perderse en el anonimato local o regional, en el ensimismamiento que cada investigador procura. Esta plataforma pretende no sólo compartir contenidos sino crear comunidad, y, en consecuencia, consolidar y enriquecer el conocimiento respecto a la Salsa como práctica cultural. 


\subsection{La falacia del inmigrante digital}

El concepto usuario se refiere a la persona que interactúa con una interfaz con la finalidad de suplir unos objetivos planteados (Carocci, 2011). Diversos han sido los autores que han abordado el tema y han propuesto una subdivisión de los usuarios (particularmente los usuarios web). Estas subdivisiones se basan principalmente en la frecuencia de uso del sistema (Eason, 1987), en el grado o el tipo de implicación en un proyecto (Cotterell \& Hughes 1995), en el uso directo o indirecto del sistema (Newman \& Lamming 1995), en el rol en la proyectación, en el desarrollo y la utilización del sistema (Sutcliffe, 2000). Sin embargo la clasificación sigue estando basada en la experticia del usuario. Por ejemplo la clasificación planteada por Prensky (2001) en la que los usuarios se clasifican en inmigrantes y nativos digitales, sostiene que los nativos son aquellos nacidos después de los noventa y los inmigrantes son aquellos que nacieron antes de la generalización de entornos digitales y tendrían marcadas diferencias.

Este término, de moda en los últimos años, no tenía en Prensky el sentido absoluto y taxativo con que se usa hoy. Prensky pensaba el término aludiendo a las diferencias que solemos encontrar entre el tipo de interacción de nuestros abuelos y el de los niños en relación con las tecnologías. A manera de chis destacaba el sentido de una afirmación coloquial muy frecuente: «la tecnología me atropella». Esta declaración representaría el tipo de comportamientos típicos de ciertos usuarios. Pero Prensky no llegó nunca a imaginar que su manera de ver este fenómeno terminara asumiéndose como una verdad absoluta, sin atender la reflexión que entrañan sus afirmaciones y planteamientos.

Otros como Shneiderman \& Plaisant (2006) y Nielsen (1993) son menos osados y se quedan con la categorización simple que distingue en tres niveles a los usuarios (básicos, intermedios y avanzados).
Con este planteamiento no se comprometen más allá de la pericia que puede demostrar un usuario frente al uso de los dispositivos o aplicaciones y no se ocupan demasiado de caracterizar la heterogeneidad de los usuarios en sí mismos.

Por su parte Tapscott (2009) propone algo más revolucionario que los autores anteriores y critica la forma monocromática de definir los usuarios por parte de Prensky. El autor propone lo que se conoce como Generación Red, intentando explicar las variaciones de usuarios que se han criado a la par de los cambios tecnológicos, y poniendo énfasis en algunas de sus características: aspiración a la libertad de elección y expresión, inclinación por la personalización y por customizar los bienes que usan, interés por el juego y el trabajo colaborativo, valoran la velocidad y la innovación, entre otros rasgos.

Los anteriores autores han estudiado con detalle el asunto relacionado con la problemática de los usuarios. No se puede afirmar que son los únicos pero sí los más reconocidos a nivel mundial.

Entendiendo este fenómeno como un problema de interpretación, pensar en las particularidades de los usuarios se convierte en un problema que atañe a todos los proyectos que involucren usuarios (proyectos de investigación y creación). De esa manera, resolver la adaptatividad de las interfaces es un tema que cobra fuerza, aunque en la actualidad, está más en función de los dispositivos que de los usuarios (precisamente por sus grados de heterogeneidad). Para resolverlo se ha tomado el estudio de Lin (2013) quien propone una clasificación de usuarios más ajustada a lo real, y no deja pie a las superficialidades mediáticas que suelen ponerse de moda. Es una forma un tanto justa de clasificar a los usuarios y no deja de lado a las personas que tienen un nivel "alto" o "bajo", de acuerdo con los estándares propuestos por Shneiderman. 
Lin (2013) clasifica y categoriza los usuarios de acuerdo con el siguiente esquema:

1. Mesurado: Este tipo de usuario realiza una conceptualización abstracta del contenido, es decir una paneo general de la información que el dispositivo ofrece. Además la experiencia de interacción es concreta, lo que significa que realiza en lo posible una actividad por vez y prefiere tomarse el tiempo necesario para realizar una tarea.

2. Observador: Realiza un paneo general de la información que el dispositivo ofrece igual que el anterior, sin embargo hace una observación más reflexiva del contenido intentando encontrar elementos que le permitan hacer relaciones que favorezcan la recordación de los procesos.

3. Orientado a Objetivos: Al igual que el anterior, realiza un paneo general de la información dispuesta pero realiza una experimentación activa con respecto a la información presentada en el dispositivo.

4. Arriesgado: Realiza una experimentación activa y directa con respecto a la información pero su experiencia se realiza en torno a actividades concretas y no tan genéricas, es decir realiza una actividad por vez con la finalidad de buscar indicios que permitan mejorar su recordación.

5. Pionero: Realiza una experiencia concreta con respecto a la información, es decir el paneo se hace de manera genérica pero centralizada en la tarea que se quiere desarrollar y hace una observación reflexiva del entorno intentando buscar relaciones basadas en conocimientos previos.

En la tabla 1 se observan las características generales que implica cada tipo de usuario.

\begin{tabular}{|l|c|}
\hline \multicolumn{1}{|c|}{ Tipo de Usuario } & Características \\
\hline Mesurado & Conceptualización abstracta y experiencia concreta. \\
\hline Observador & Conceptualización abstracta y observación reflexiva. \\
\hline Orientado a Objetivos & Conceptualización abstracta y experimentación activa. \\
\hline Arriesgado & Experiencia concreta y experimentación activa. \\
\hline Pionero & Experiencia concreta y observación reflexiva. \\
\hline
\end{tabular}

Tabla 1. Usuarios y sus características. Lin (2013). 
Por medio de la observación directa en el tiempo de ejecución del proyecto pudimos percatarnos que todos los tipos de usuarios están presentes en los espectadores del proyecto, sin embargo quisimos proponer una propuesta de interfaz que si bien es cierto utiliza un conjunto de herramientas generadas a partir de HTML5, no están saturadas de animaciones y transiciones lo cual evita la sobrecarga cognitiva de los usuarios y permite una concentración en el contenido. Todos estos elementos son insumos fundamentales para concretar las determinantes particulares del diseño de la interfaz. Por otro lado la necesidad de mostrar unos ires y venires considerados por el profesor Alejandro Ulloa como partes cruciales del proceso de migración y de la diáspora en si misma de la música afrolatinocaribeña a nivel mundial, nos indicó la importancia de plantear esos lugares en tanto representación de la realidad sin ser un calcos exactos (Reyes \& Becerra, 2011). Esto hizo que concibiéramos en el proyecto una serie de lugares ficticios como metáfora de navegación e interacción en los que cada uno de los elementos compositivos debía ser pensado en función del contexto (de una época y de un lugar). Se mantuvo la preocupación de dar a conocer esos contenidos no sólo a nivel de texto sino audiovisual con el objetivo de permitirle al espectador reconocer de manera más directa el contexto de los contenidos y permear de forma directa o indirecta todos los tipos de usuarios planteados en la tabla 1.

\section{La adaptatividad, adaptabilidad y sensibilidad al contexto}

Según Mobasher (2007) la adaptabilidad está relacionada con la personalización que puede darle un usuario a una interfaz, de tal forma que consiga ajustar algunas propiedades interactivas para mejorar su desempeño a partir de la subjetivización de los elementos. Por otra parte, la adaptatividad se relaciona con las posibilidades que tiene el sistema de auto configurarse según las necesidades intrínsecas del usuario, mejorando las cualidades prestacionales que brindan los elementos interactivos.

Existen muchos ejemplos web que ofrecen adaptabilidad y adaptatividad. Un ejemplo del primero es un sitio de e-comerce como Dell (www.dell.com) que permite a los usuarios del sistema manipular ciertas configuraciones manualmente antes de decidirse por una compra. Un ejemplo del segundo es Amazon (www.amazon.com) que genera recomendaciones de diversos productos de acuerdo con un perfil particular de usuario y sus intenciones de compra mediado también por compras ya realizadas.

Dada la diversidad de dispositivos y plataformas es muy difícil pensar en una interfaz que sea estática y que se presente a todos los usuarios de igual manera. Por esa razón ha surgido lo que se conoce en el argot del diseño web como Responsive Web (URL2) que no es otra cosa que una interfaz web sensible al contexto y que se adapta de manera automática a los diversos dispositivos.

Esta es una preocupación que surgió en el planteamiento del proyecto de creación, pues con la tecnología que se implementó la Multimedia Cali Cultura Salsera era difícil manejar este aspecto, por esa razón era una aplicación netamente pensada para un computador de escritorio y dejaba de lado la posibilidad de realizar una interacción de los usuarios con 
dispositivos móviles (siendo hoy cada vez más comunes y de bajo costo). Eso sin contar con la dificultad que tenía la plataforma de streaming de video que le impedía a los usuarios la observación del material sin tener que someterse a la latencia de la descarga de los archivos y la velocidad de la red.

Desde el punto de vista técnico la Hipermedia Salsa Barrio Cultura es mucho más versátil y de fácil mantenimiento, pues sus contenidos pueden actualizarse de manera más eficiente que con otras plataformas. Desde el punto de vista estético tiene unas prestaciones particulares que se explicarán posteriormente. Pero lo que más importa es la manera como se simplifica la interfaz en los dispositivos móviles y le permite al usuario interactuar con la información del modo más sencillo posible.

A continuación se muestra la interfaz de la Hipermedia Salsa Barrio Cultura en tres diferentes tipos de dispositivos en donde se analizarán las diversas variaciones a nivel morfológico y estructural de la interfaz.

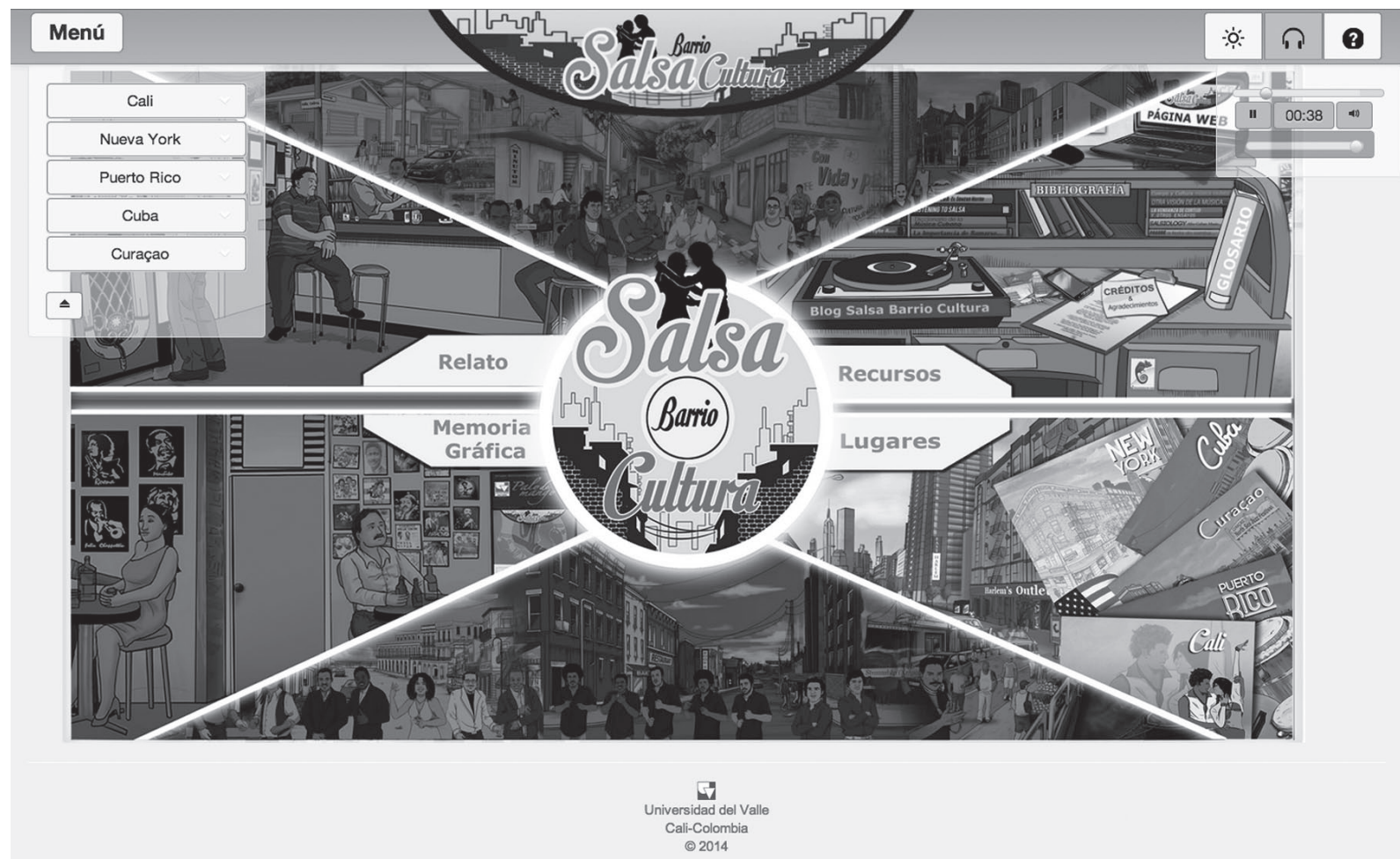

Figura 1. Disposición de los elementos de la Interfaz en resolución 1360x768 px Laptop. 
Figura 2. Disposición de los elementos de la Interfaz en resolución $320 \times 480$ px iPhone.

Como se observa en las Figuras 1 y 2, la misma interfaz se modifica de acuerdo con el tamaño del dispositivo, no sería adecuado dejar la misma interfaz en los dos dispositivos, ya que en los móviles se perderían detalles y el usuario no podría interactuar de manera adecuada con los gráficos de fondo. Por otra parte, la interfaz respeta la consistencia y mantiene la coherencia visual de las interfaces adaptativas a pesar de que visualmente tengan un cambio considerable en su composición.
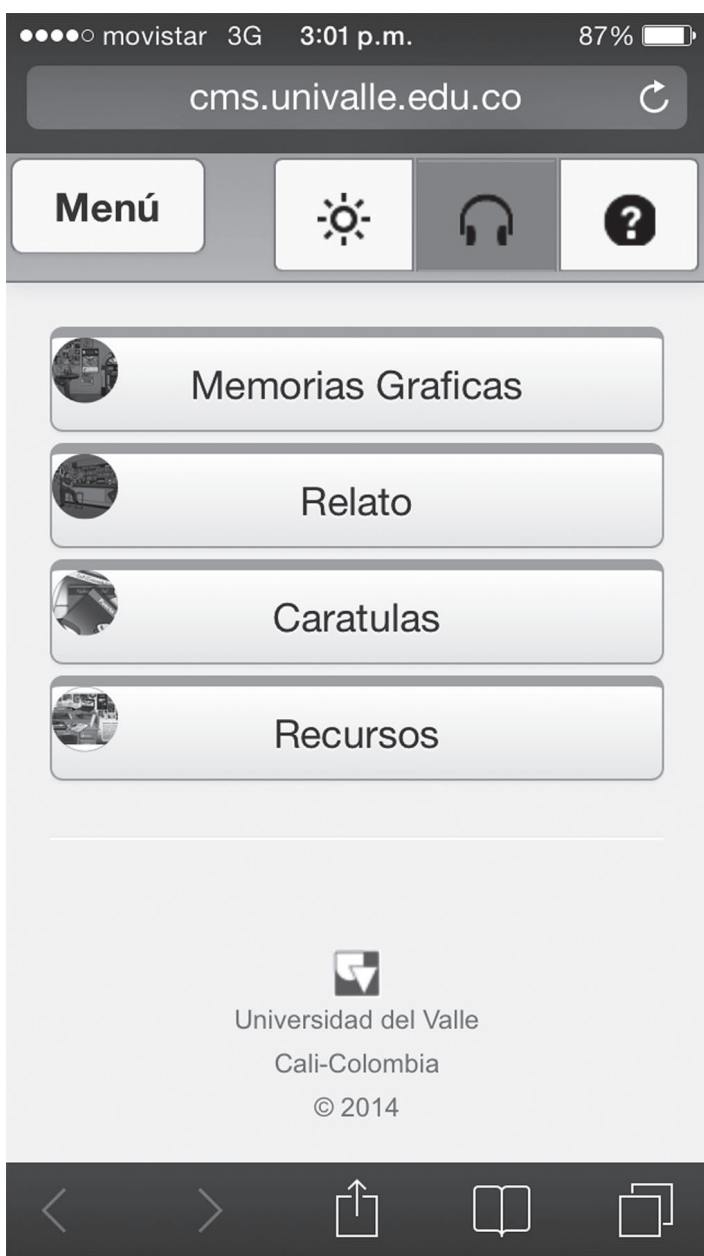

\section{Buscando una estructura narrativa}

Literalmente la usabilidad es la capacidad de un artefacto de ser usado, pero esta expresión sustenta un concepto más amplio en el que cabe afirmar que no basta con que sea usado, sino bien usado, es decir, sin dificultad. Este término fue propuesto por Bennett (1979) para identificar aquello que hace que un dispositivo sea conveniente y práctico para un usuario. Cada sistema debe ser fácil de aprender y de recordar, ser usable y contener funciones que realmente sirvan. De acuerdo con Gould y Levis (1985) existen tres principios fundamentales para una buena proyectación de una interfaz:

1. Focalizarse en los usuarios y las tareas que debe desarrollar con el sistema.

2. Utilizar medidas empíricas

3. Adoptar una lógica iterativa

Con base en esos principios se considera que una interfaz debe tener las cualidades mínimas que se espera desde el punto de vista de la usabilidad y que puede ofrecer al usuario un conjunto de elementos con los que interactuar de manera directa y contextualizada. 
Diseñar interfaces en las que se requiera compartir información implica pensar una estructura lógica y coherente que permita organizar secuencialmente los contenidos, no se trata simplemente de disponerlos en un cascarón; sino de que le permitan al usuario encontrar relaciones y hacer inferencias respecto a la información contenida. Otra cosa que se busca es dejar pie para fomentar la autonomía, que el usuario sienta la curiosidad de seguir explorando el tema.

Por eso la información debe estar organizada y estructurada de modo tal que el usuario perciba una calidad que permita granularizar esas relaciones. El equipo de trabajo del proyecto de creación se esmeró por darle un sentido a los elementos compositivos de la interfaz: la pregunta constante era ¿por qué? Así se desarrolló un conjunto de estructuras que permitieron cotejar las limitaciones y alcances de la técnica con la riqueza de la interfaz en lo atinente a sus aspectos estéticos y funcionales.

En la Hipermedia Salsa Barrio Cultura se proponen varias metáforas que se integran en un solo discurso. No se ha dejado ningún elemento al azar, por ello puede afirmarse que la creación en sí misma es fruto de la reflexión colegiada del grupo de trabajo.
Por esa razón tanto representación de lugares, personajes y elementos de contexto, personajes principales y acciones de manipulación de los elementos de la interfaz están pensados de tal manera que se integren sinérgicamente para lograr en el espectador los objetivos que busca, sin incurrir en esfuerzos cognitivos innecesarios que puedan generar abandonos de la aplicación. Tratamos de supeditar la tecnología Html5 a las necesidades de proyectación y se plantearon retos importantes a nivel de implementación. Se alcanzaron resultados inéditos que pueden ser tomados como punto de referencia en futuros desarrollos o creaciones similares.

Si bien es cierto que metodológicamente en el proyecto anterior se trabajó con las mismas premisas a nivel estructural, los alcances del proyecto Hipermedia Salsa Barrio Cultura han sido mucho más robustos en términos de implementación de contenidos y de programación mutimodal y multimedial.

A continuación se muestran algunas de las estructuras narrativas evidentes en el proyecto:

1. Metáforas de carátulas de discos respecto a los lugares.
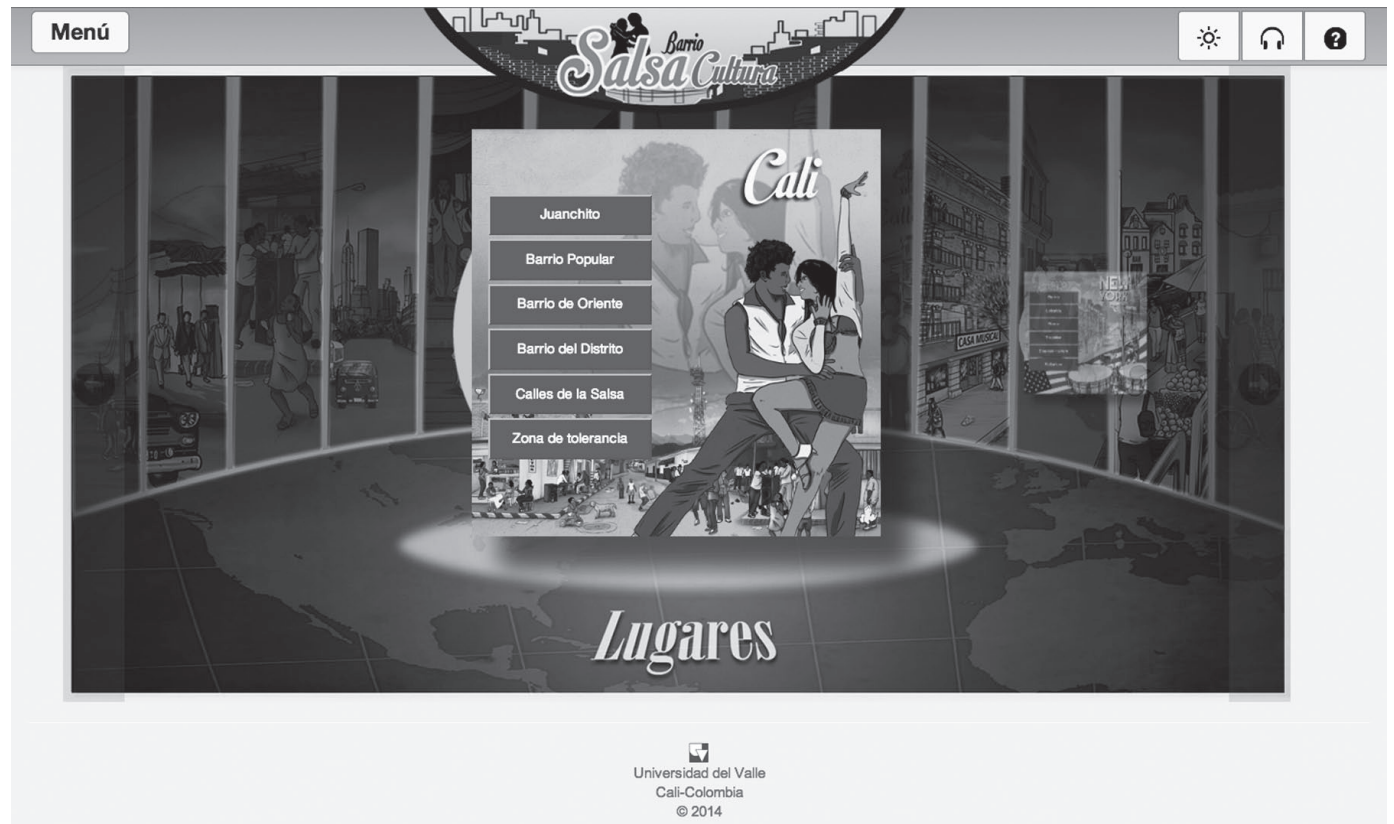
Las carátulas tienen el esquema de carrusel en el que se van moviendo gradualmente de derecha a izquierda ya sea presionando los botones laterales o moviéndolas con los dedos (para dispositivos tipo touch screen). Esta metáfora le permite al usuario encontrar los lugares que contiene la Hipermedia de una manera más directa, pues al presionar en las etiquetas de cada lugar surgen las diversas opciones que se despliegan como un disco, como se puede apreciar en la siguiente imagen.

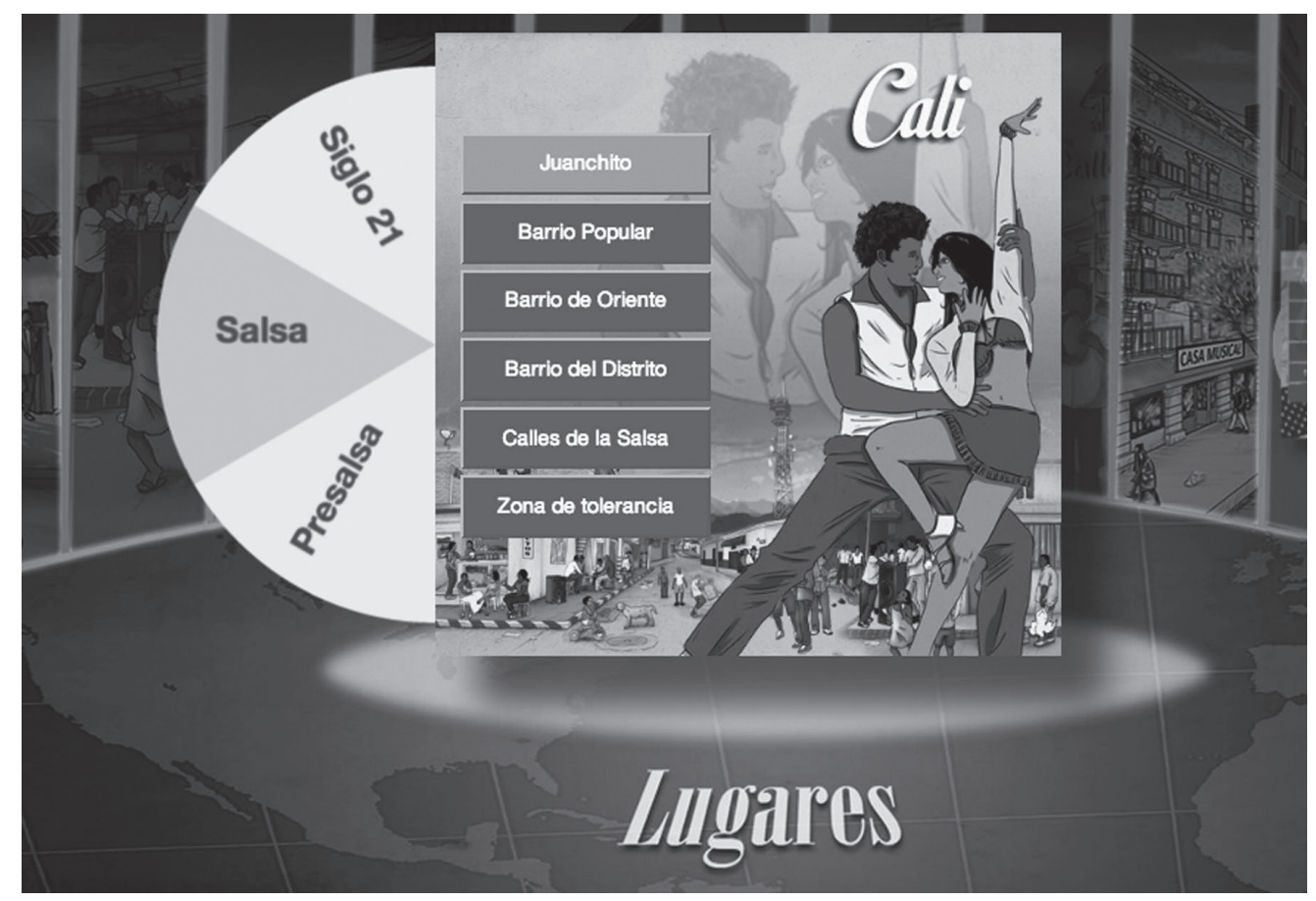

\section{Apreciación espacio- temporal de lugares ficticios}

Como ya se enunció, los lugares se han creado siguiendo una linealidad relacionada con la información que componen los años de investigación de la Salsa. De esa forma, se definen y se crean a partir de la transmutación mimética de diversos elementos compositivos. No se busca representar fielmente el lugar sino colocar al usuario en un lugar ficticio con las características del sitio al que evoca. Es importante destacar que los lugares ficticios contienen elementos y personajes de contexto que fungen como mediadores entre la percepción del lugar y del tiempo. En este sentido se observan mascotas, banderas, afiches, grafitis, personas, autos, etc., que permiten realizar una inferencia más directa del lugar que representan y refuerzan el discurso.

A continuación se muestran dos de esos lugares ficticios y se explicará brevemente qué contienen y las razones por las cuales se los diseñó de esa forma. 


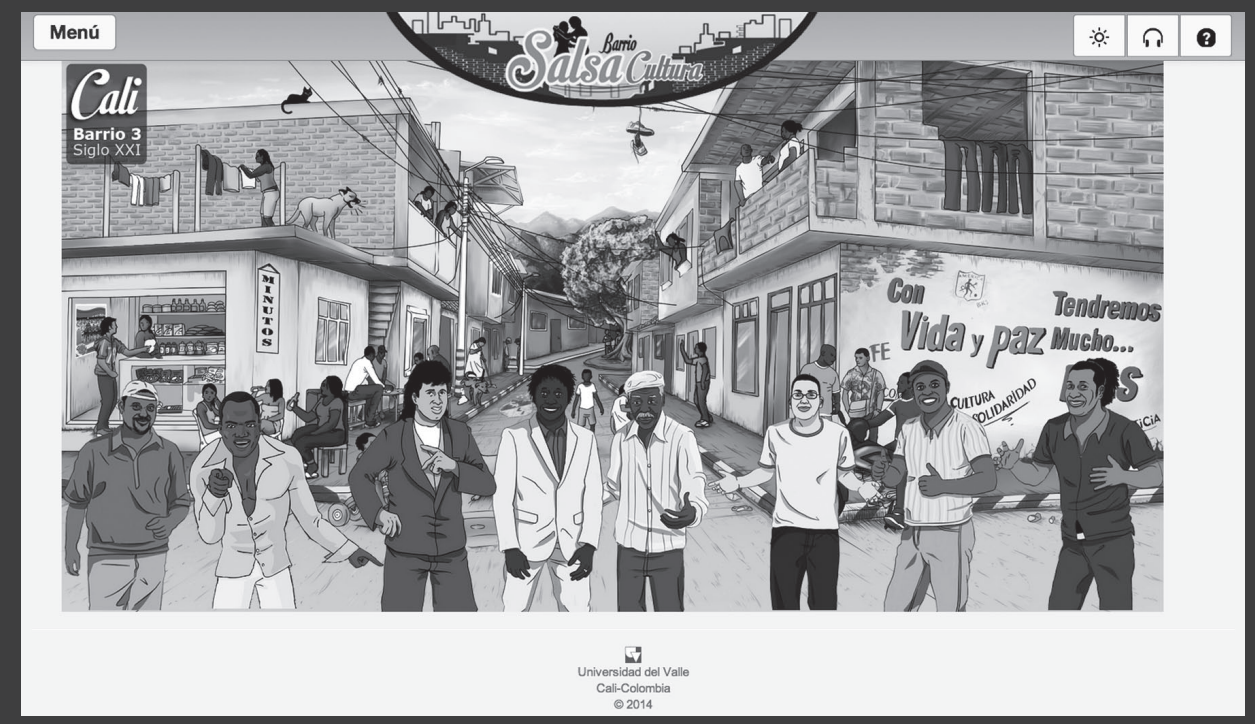

Este lugar corresponde al barrio popular en Cali, quizás una representación de lo que se conoce como el sector de Agua Blanca. Aquí se aprecian algunos grafitis que hacen parte el entorno cotidiano de la violencia barrial que se vive en nuestra ciudad; también la tienda popular de barrio en la que se observa el aviso de minutos de celular (una práctica muy común en Colombia y en nuestro siglo). Teniendo en cuenta que esta imagen podría ser una representación de cualquier barrio se han incluido los escudos del Cali y del América, estos elementos sumados con las etnias de los personajes de contexto, refuerzan el discurso buscado.

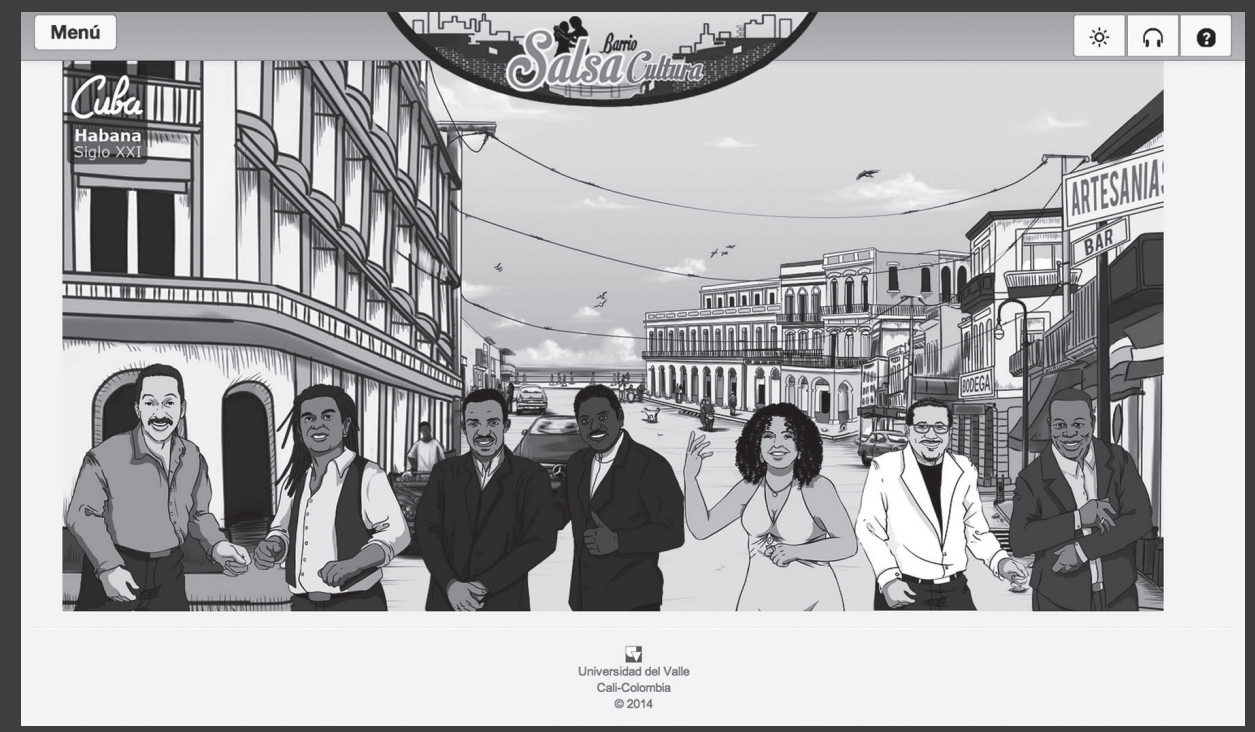

Otro lugar ficticio emblemático es La Habana en el Siglo XXI en la que se observa el Malecón, algunas gaviotas que refuerzan la sensación de una ciudad costera, pero además la arquitectura particular de la isla. Desde luego los personajes de contexto permiten ubicar al usuario en la época por su vestido y el desparpajo propio del cubano. 
Así como estos dos ejemplos los demás fondos ficticios proponen elementos que permiten darle coherencia a todo el discurso de la creación.

\section{La hipertextualidad: un elemento base}

Desde luego la hipertextualidad es una herramienta que permite navegar en contenidos de forma secuencial y controlada, pues combina las características intrínsecas de la multimedia (texto, audio e imagen -estática o en movimiento-) con la hipertextualidad que le permite al usuario saltar de nodo a nodo de información a voluntad (Díaz, Catenazzi \& Aedo, 1996; Balasubramanian, 1995; Rada, 1991).

La conjugación adecuada de estos elementos permite tener mucha información en poco espacio: el resultado son interfaces menos saturadas, lo cual favorece la interacción con la información presentada y, en el usuario, una interrelación controlada.

A continuación se muestra cómo se manifiesta la hipertextualidad en la obra tanto para dispositivos móviles como para computadores de escritorio.

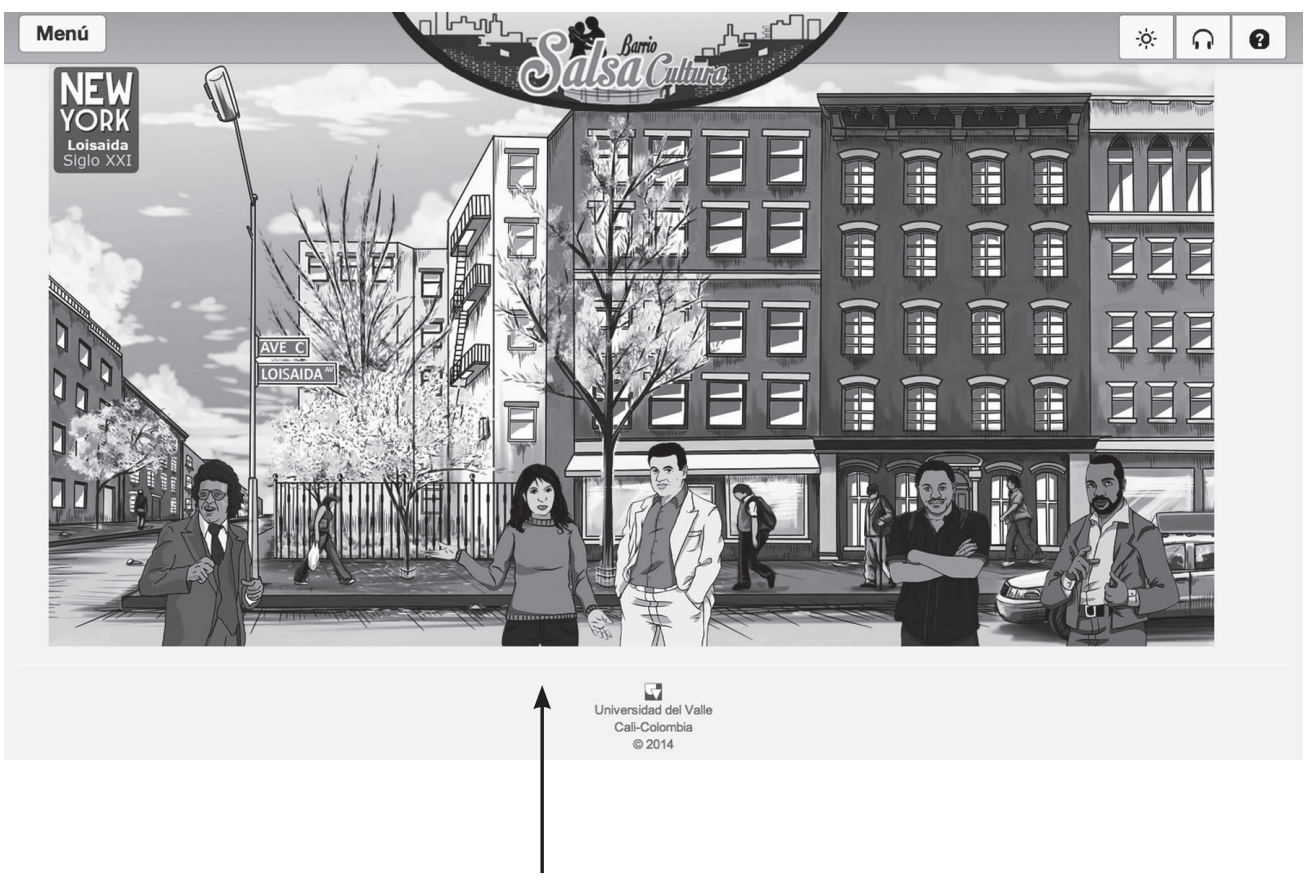

Para este ejemplo el usuario ve la interfaz sin acción alguna y decide presionar el ratón sobre el personaje de Aurora Flores (mujer de rojo). 
Una vez lo hace carga el contenido respectivo.

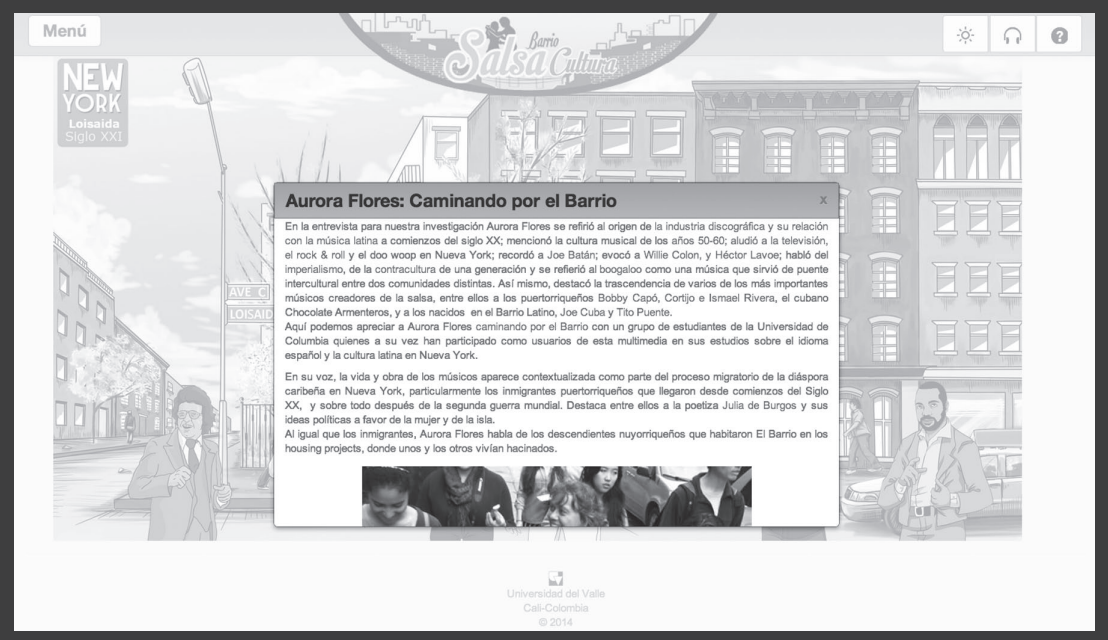

Puede interactuar con este contenido a través del scroll, con la rueda central del ratón o con sus dedos (en caso de que tenga pantalla touch screen).

Si selecciona algún texto que está en otro color va a cargar un video relacionado.

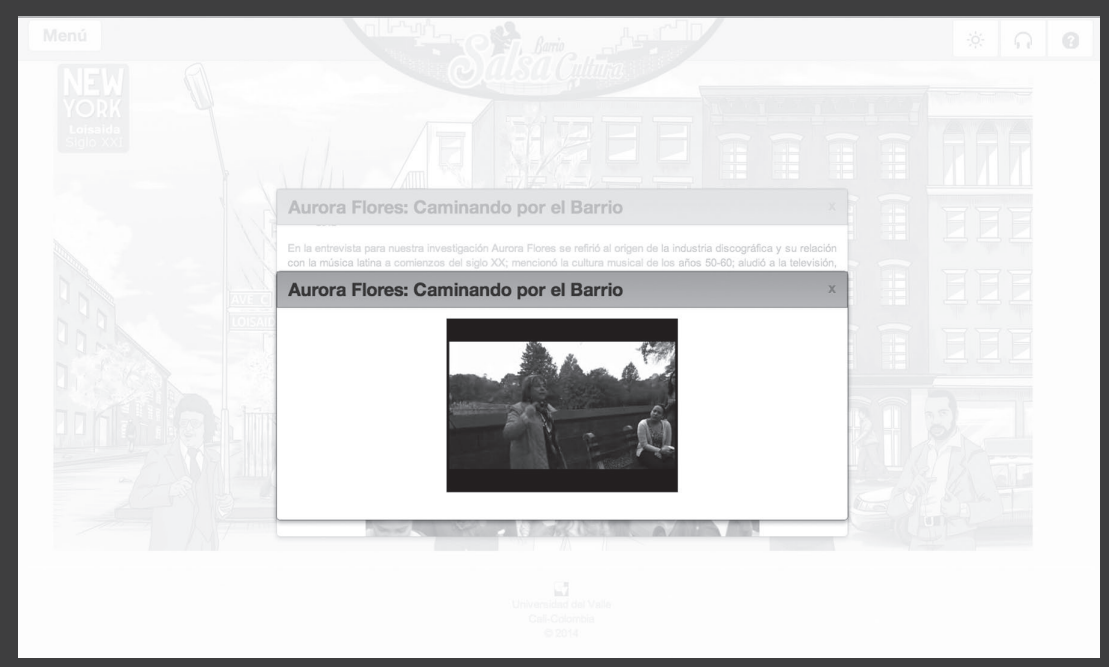

Versión Móvil:

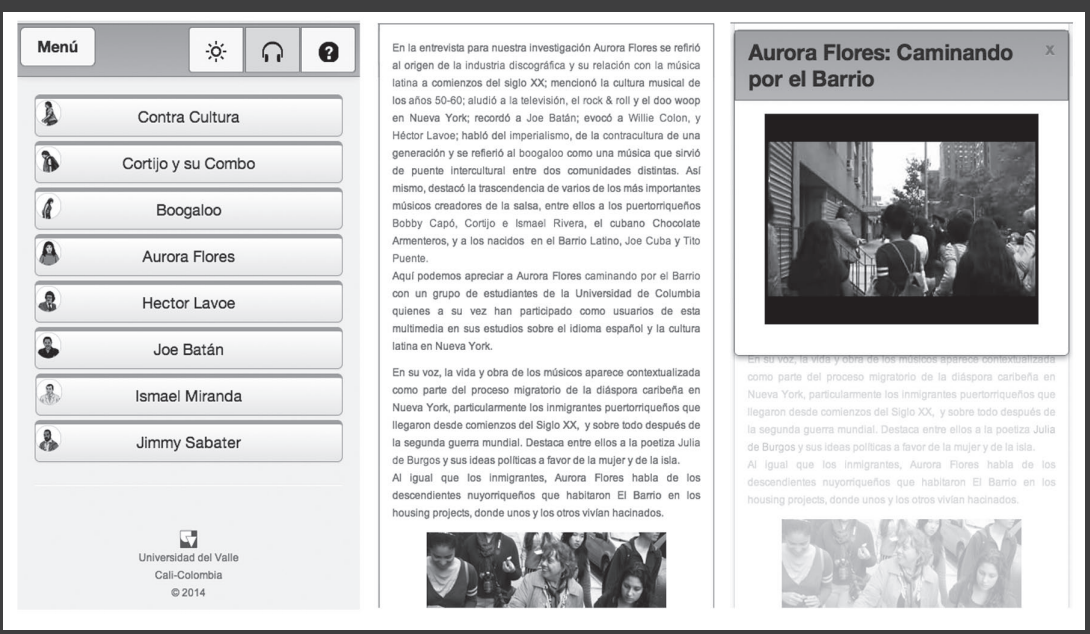


Como se observa, la interfaz en los dos dispositivos presentados genera una opacidad en el estado anterior, pero no cierra la ventana, lo cual le permite al usuario continuar en contexto y, una vez haya observado el video (que tiene la opción de darle pantalla completa), lo cierra y puede continuar la lectura.

\section{Conclusiones}

La creación de la interfaz de una hipermedia adaptativa implica no sólo considerar las limitaciones técnicas y tecnológicas, también las particularidades de los usuarios con la finalidad de proponer elementos estéticos ajustados a la narrativa propuesta. En este sentido las metáforas propuestas deben garantizar la integralidad del discurso.

Html5 permite visualizar la Hipermedia en diversos dispositivos, lo cual facilita la ampliación de los públicos diversos y permite que accedan a los contenidos de manera más frecuente. Los aspectos técnicos y tecnológicos en el desarrollo de un proyecto de creación no deben ser limitantes a la hora de abordar elementos estéticos, por el contrario deben estar su servicio, por esta razón es necesaria una perfecta sincronía en el grupo de trabajo para que todos los actores que participan entiendan el trabajo y los resultados satisfagan las expectativas de calidad a que aspiran obras de esta magnitud.

La Hipermedia Salsa Barrio Cultura es única en su género, pues no existe un producto que se le compare en cuanto a su complejidad estructural y compositiva. En ella se han integrado efectivamente aspectos de orden etnográfico, estéticos y de interfaz en un producto cuidadosamente desarrollado por un grupo interdisciplinario de estudiantes, asistentes, técnicos y profesores de la Universidad del Valle.

\section{Notas}

${ }^{1}$ Proyecto aprobado por la convocatoria interna para proyecto de Creación de la Vicerrectoría de investigaciones de la Universidad del Valle año 2012 en el que participaron los grupos de investigación Palo de Mango y Camaleón de la Universidad del Valle. Para mayor información de este proyecto http: / / salsabarriocultura.univalle.edu.co

${ }^{2}$ De acuerdo con Bush, el Memex es un almacenamiento conceptual y un sistema de recuperación de datos. Este concepto fue concebido para ayudar a las personas a mejorar sus vidas mediante el acceso a la enorme cantidad de conocimiento ya almacenada. El autor se percató de que no existía una manera sencilla de navegar en esta información y también encontrar o relacionar esa información una con otra. Precisamente la idea de memex estaba encaminada a solucionar estas falencias.

${ }^{3}$ Un ejemplo concreto de este punto son las redes sociales, donde muchas personas generan niveles de empatía más directos que en la vida cotidiana, o el uso e blogs, wikis y otros elementos que sacan del anonimato a una persona del común y lo lanzan al estrellato. 


\section{Referencias}

Balasubramanian, V. (1995). State of the art review of hypermedia: issues and applications.

Bartlett, F. (1962). Remembering, Cambridge University Press. The Future of Ergonomics in Ergonimics, pp. 505-11.

Bennett, J. L. (1979). The Commercial Impact of Usability in Interactive System, in B. Shackel ed. Man/Computer Communication, Infotech, Maidenhead, Vol. 2, pp. 1-17.

Bush, V. (1945). As we may think. En: The Atlantic monthly, 1945, n. 176, pp. 101-108.

Carocci, (2011). Ergonomia cognitiva. A cura de Francesco di Nocera. Carocci ed.

Cotterell, M. \& Hughes, B. (1995). Software Poject Managment. International Thompson Publishing, London.

Díaz, P., Catenazzi, N. \& Aedo, I. (1996).De la multimedia a la hipermedia. Madrid, Editorial Rama.

Eason,K. (1987). Information Technology and Organizational Change. Taylor \& Francis. London.

Engelbart, D. (1962). Augmenting human intellect: a conceptual framework. Stanford Research Institute.

Feurzeing, W. (1966). The LOGO Lineage. The Logo foundation.

Gould, J. \& Lewis, C. (1985). Designing for Usabiltity: Key principals and what Designers Think. Communications ACM. 28(3), pp. 300-311.

Grudin, J. (1990). The Computer Reaches Out: The historical Continuity of Interface Design. SIGCHCI. Conference on Human Factors in Computing Systems: Empowering People. ACM press NY pp.261-8.

Licklider, J. \& Carl R. (1990). Man-computer symbiosis. En: In memoriam:J. C. R. Licklider 1915-1990. Palo Alto, California: Digital Systems Research Center, 1990.

Lin, T. (2013) Attraction and Action. User types blending aesthetical and functional design. IEEE 2013.

Mobasher, B. (2007) Data Mining for Web Personalization. The Adaptive Web, LNCS 4321, pp. 90-135.

Myers, B. (2005). A brief history of human-computer interaction technology. En: Interactions, 1998, v. 5, n. 2, pp. 44-54.

Nelson, T. (1965) A tile structure for the complex, the changing and indeterminate. ACM national conference, pp. 84-100.

Newman, W. \& Lamming, M. (1995). Interactive System Design, Addison Wesley.

Nielsen, J. (1993). Usability Engineering. 1ra Ed. United States of America: Academic Press, 1993. ISBN-10:0-12-518406-9. Prensky (2001). Digital Natives, Digital Immigrants. From On the Horizon (MCB University Press, Vol. 9 No. 5, October 2001).

Rada, R. (1991): Hypertext: from text to expertext. McGraw-Hill.

Reyes, J. \& Becerra, E. (2011) Escenarios y personajes en la "Multimedia Cali Cultura Salsera”. Revista Nexus, v 10 pp p. $190-203$.

Ribera, M. (2005). Evolución y tendencias en la interacción persona-ordenador. El profesional de la información, v. 14, n. 6, noviembre-diciembre 2005.

Shackel, B.(1997). Human-computer interaction: whence and whither. En: Journal of the American Society for Information Science, v. 48, n. 11, pp. 970-986.

Shneiderman, B.(1987) Designing the user interface: strategies for effective human-computer interaction. Reading, Massachusetts: Addison-Wesley.

Shneiderman, B \& Plaisant, C.(2006). Designing the user interface: strategies for effective human-computer interaction. 4 a ed. Boston: Pearson Education.

Sutcliffe, A. (2000). User-Centered Requirements Engineering:Theory and Practice. Springer. New York.

Tapscott. D. (2009) La era digital. Cómo la generación net está transformando al mundo. McGraw Hill, México

Weiser, M. (1991). The computer for the twenty-first century». En: Scientific American, 1991, pp. 94-104.

World Wide Web Consortium, W3C multimodal interaction activity.

Consultado en: 15-04-14. http://www.w3.org/2002/mmi/URL1 http://caliculturasalsera.univalle.edu.co

URL2 http:/ / www.princeton.edu/ mlovett/reference/A\%20List\%20Apart-Articles-Responsive\%20Web\%20Design.pdf

Recibido: abril 15 / Aprobado: junio 1 de 2014 